\title{
DNA Methylation and Protein Markers of Chronic Inflammation and Their Associations With Brain and Cognitive Aging
}

Eleanor L.S. Conole, BSc, MRes, Anna J. Stevenson, PhD, Susana Muñoz Maniega, PhD, Sarah E. Harris, PhD, Claire Green, MSc, Maria del C. Valdés Hernández, PhD, Mathew A. Harris, PhD, Mark E. Bastin, DPhil, Joanna M. Wardlaw, MD, Ian J. Deary, PhD, Veronique E. Miron, PhD, Heather C. Whalley, PhD, Riccardo E. Marioni, PhD, and Simon R. Cox, PhD

Neurology ${ }^{\circledR}$ 2021;97:e2340-e2352. doi:10.1212/WNL.0000000000012997

\section{Abstract}

\section{Background and Objectives}

To investigate chronic inflammation in relation to cognitive aging by comparison of an epigenetic and serum biomarker of C-reactive protein and their associations with neuroimaging and cognitive outcomes.

\section{Methods}

At baseline, participants $(n=521)$ were cognitively normal, around 73 years of age (mean 72.4, SD 0.716), and had inflammation, vascular risk (cardiovascular disease history, hypertension, diabetes, smoking, alcohol consumption, body mass index), and neuroimaging (structural and diffusion MRI) data available. Baseline inflammatory status was quantified by a traditional measure of peripheral inflammation - serum C-reactive protein (CRP) - and an epigenetic measure (DNA methylation [DNAm] signature of CRP). Linear models were used to examine the inflammation-brain health associations; mediation analyses were performed to interrogate the relationship between chronic inflammation, brain structure, and cognitive functioning.

\section{Results}

We demonstrate that DNAm CRP shows significantly (on average 6.4-fold) stronger associations with brain health outcomes than serum CRP. DNAm CRP is associated with total brain volume $\left(\beta=-0.197,95 \%\right.$ confidence interval $[\mathrm{CI}]-0.28$ to $\left.-0.12, p_{\mathrm{FDR}}=8.42 \times 10^{-6}\right)$, gray matter volume $\left(\beta=-0.200,95 \% \mathrm{CI}-0.28\right.$ to $\left.-0.12, p_{\mathrm{FDR}}=1.66 \times 10^{-5}\right)$, and white matter volume $\left(\beta=-0.150,95 \% \mathrm{CI}-0.23\right.$ to $\left.-0.07, p_{\mathrm{FDR}}=0.001\right)$ and regional brain atrophy. We also find that DNAm CRP has an inverse association with global and domain-specific (speed, visuospatial, and memory) cognitive functioning and that brain structure partially mediates this CRP-cognitive association (up to $29.7 \%$ ), dependent on lifestyle and health factors.

\section{Discussion}

These results support the hypothesis that chronic inflammation may contribute to neurodegenerative brain changes that underlie differences in cognitive ability in later life and highlight the potential of DNAm proxies for indexing chronic inflammatory status.

\author{
Correspondence \\ Ms. Conole \\ eleanor.conole@ed.ac.uk
}

\section{MORE ONLINE}

\section{Class of Evidence}

Criteria for rating therapeutic and diagnostic studies

NPub.org/coe 


\section{Glossary}

$\mathbf{A D}=$ Alzheimer disease $\mathbf{B M I}=$ body mass index; $\mathbf{C F I}=$ comparative fit index; $\mathbf{C I}=$ confidence interval; $\mathbf{C R P}=\mathrm{C}$-reactive protein; CVD = cardiovascular disease; $\mathbf{d M R I}=$ diffusion MRI; DNAm = DNA methylation; EWAS = epigenome-wide association studies; FDR = false discovery rate; $\mathbf{g f}$ = general cognitive ability; $\mathbf{g F A}=$ general fractional anisotropy; $\mathbf{G M}=$ gray matter; gMD = general mean diffusivity; ICV = intracranial volume; NAWM = normal-appearing white matter; $\mathbf{Q C}=$ quality control; RMSEA = root mean squared error approximation; SRMR = standardized root mean square residual; $\mathrm{TB}=$ total brain; TLI = Tucker-Lewis index; VRF = vascular risk factor; $\mathbf{W M}=$ white matter; $\mathbf{W M H}=$ white matter hyperintensity.

\section{Classification of Evidence}

This study provides Class II evidence that a DNAm signature of CRP levels is more strongly associated with brain health outcomes than serum CRP levels.

Low-level systemic chronic inflammation has emerged as a hallmark and potential driver for individual differences in brain aging. ${ }^{1-5}$ Yet while chronic inflammation has been consistently linked to dementia, ${ }^{6-8}$ studies investigating peripheral inflammatory markers in nonclinical groups show disparity with respect to cognitive outcomes ${ }^{9-14}$ and have not yet clarified the magnitude and regional extent of brain structural associations. ${ }^{13-18}$

One reason for this inconsistency is that there are no standard biomarkers for chronic inflammation, and to date many studies have relied upon blood biomarkers of acute inflammation such as C-reactive protein (CRP). A significant caveat of this approach is assuming baseline inflammation status from highly phasic protein levels, which are subject to swift and rapid concentration changes in blood plasma. ${ }^{19}$ This introduces significant noise at the epidemiologic level ${ }^{20,21}$ (Figure 1D) and few studies take repeat measures of serum $\mathrm{CRP}^{12}$ or attempt to correct for within-person fluctuations. A more accurate reflection may come from an epigenetic approach: DNA methylation (DNAm) profiles have been identified in inflammatory diseases ${ }^{22,23}$ and inflammationrelated disease outcomes ${ }^{24,25}$ and are theorized to provide more stable reflections of inflammatory exposure. ${ }^{24,26-28}$ In the same cohort as in the present study, a DNAm proxy of CRP exhibited greater longitudinal stability and stronger associations with cognitive functioning than serum CRP levels. ${ }^{11,28}$

Here, we predict that a DNAm signature of CRP will show significantly stronger associations with brain health outcomes than its serologic counterpart. Our objective is to examine the

Figure 1 Chronic Inflammation Increases With Age and May Contribute to Variance in Cognitive Ability

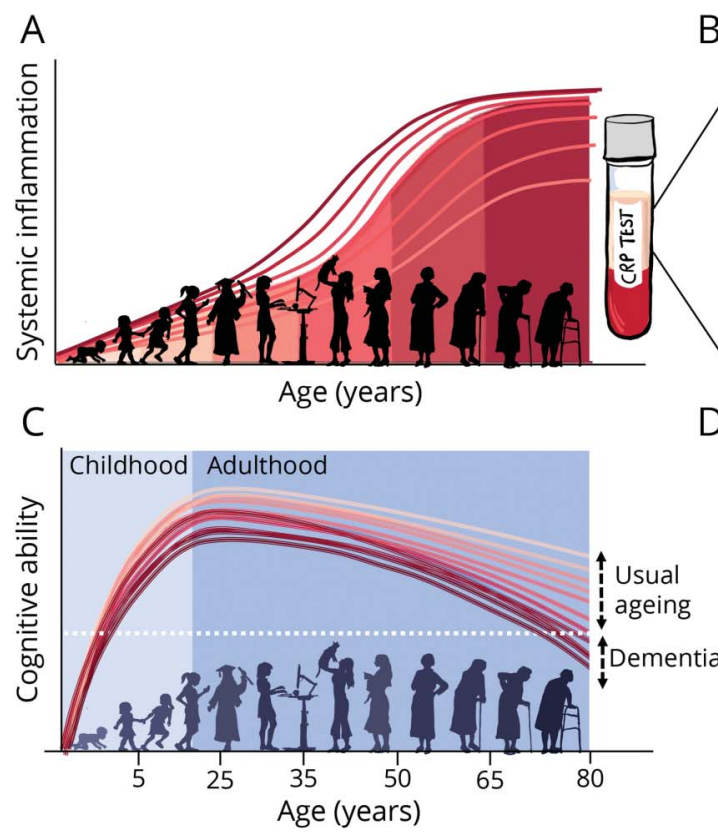

B
(A) Schematic demonstrating lifespan curves for chronic inflammation. Inflammatory load tends to increase with age; lifestyle, genetics, and health conditions can all influence susceptibility to chronic inflammation and account for variance in inflammation levels between individuals. $^{1,3}$ (B) Chronic inflammation can be measured by inflammatory proteins taken from a blood sample, such as serum levels and DNA methylation (DNAm) proxies of C-reactive protein (CRP). (C) Lifespan curves for cognitive ability outlining how there is considerable interindividual heterogeneity in rate and timing of cognitive decline, with some people on more accelerated cognitive aging trajectories than others. $^{4}$ (D) Trajectories of LBC1936 participants respective of inflammation scores over age, as outlined in Stevenson et al, ${ }^{11}$ illustrating comparative stability of DNAm inflammation marker compared to serum CRP. 
relationship between chronic inflammation, brain structure, and cognitive aging in a large community-dwelling sample of older adults.

\section{Methods}

\section{Participants}

The Lothian Birth Cohort 1936 (LBC1936) comprises individuals who were surviving members of the Scottish Mental Survey 1947, born in 1936, and who were living in Edinburgh and the surrounding area (the Lothians) when the study began in 2004. Full details of the recruitment procedures and protocols have been published. ${ }^{29}$ Participants took part in 4 waves of testing in later life (at mean ages 70, 73, 76, and 79 years) as part of an investigation into the determinants of cognitive aging. At each wave, participants were interviewed and tested individually by a trained psychologist and a research nurse during a visit to the Wellcome Trust Clinical Research Facility (wtcrf.ed.ac.uk), Western General Hospital, Edinburgh, United Kingdom. This visit included cognitive and other psychological assessments, physical examinations, extensive history taking, and blood analyses. From wave 2 onwards, neuroimaging data are also available.

The current study on chronic inflammation is cross-sectional (all variables described here were collected in 2007, at wave 2 of the LBC1936 study) and addresses the following primary research questions:

1. Does an epigenetic inflammation measure (DNAm CRP) show stronger associations with brain structure and function than serum CRP levels? (Class II evidence)

2. Does an epigenetic inflammation measure (DNAm CRP) show stronger associations with white matter (WM) microstructure than serum CRP levels? (Class II evidence)

3. To what extent can alterations in brain structure explain the association between inflammation and cognitive ability? (Class II evidence)

Participants were free from neurodegenerative diagnoses at baseline and were excluded if they had a self-reported history of stroke, Parkinson disease, or Alzheimer disease or had a Mini-Mental State Examination score <24, indicating mild cognitive impairment. We also excluded participants with serum CRP level $>10 \mathrm{mg} / \mathrm{L}$, suggestive of acute infection or illness at the time of blood draw. After exclusions, a total of 521 participants had complete inflammation, cognitive, neuroimaging, and relevant health data. For further details on data availability and attrition, see Figure 2 and eTable 1 (links.lww. com/WNL/B629).

\section{Brain Imaging Data}

Structural and diffusion tensor imaging MRI acquisition and processing in LBC1936 were performed according to an

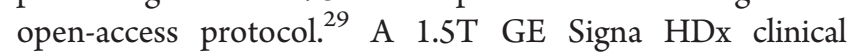

scanner (General Electric) was used to collect structural T1 (voxel size $1 \times 1 \times 1.3 \mathrm{~mm}$ ), T2 (voxel size $1 \times 1 \times 2 \mathrm{~mm}$ ), T2* (voxel size $1 \times 1 \times 2 \mathrm{~mm}$ ), and fluid-attenuated inversion recovery-weighted images (voxel size $1 \times 1 \times 4 \mathrm{~mm}$ ); for full details on MRI sequence measures, refer to Table 1 in the open access protocol article. ${ }^{29}$ Local processing and quality control (QC) of cortical reconstruction and segmentation was performed using FreeSurfer v5.1 on T1-weighted volumes. Full information on brain imaging acquisition, $\mathrm{QC}$, and variables used in analyses is detailed in the supplementary eMethods (links.lww.com/WNL/B629).

\section{CRP Data}

Serum CRP was measured from whole blood samples using a high-sensitivity assay (ELISA; R\&D Systems).

\section{DNAm Preparation and DNAm CRP Score}

Genome-wide DNAm was measured in blood samples using the Illumina Human MethylationEPIC BeadChip at the Edinburgh Clinical Research Facility Genetics Core; the epigenetic measure of chronic inflammation was calculated for each participant as described previously. ${ }^{11}$ Briefly, a DNAm CRP score was assembled for each participant in wave 2 of LBC1936; this was created by means of a weighted composite score, based on a discovery meta-analysis (9 cohorts, $\mathrm{n}=8,863$ ) and a replication meta-analysis ( 4 cohorts, $\mathrm{n}$ $=4,111$ ) of CRP-epigenome-wide association studies (EWAS). ${ }^{22}$ Methylation beta values were derived for the 7 CpG sites shown to have the strongest association with serum CRP levels, then multiplied by their standardized regression weights and added together. Given that all regression weights from the EWAS were negative, a higher DNAm CRP score (i.e., closer to 0 ) corresponds to a higher inflammatory profile. Relative weights for the $7 \mathrm{CpGs}$ are included in the supplementary document (eTable 2, links.lww.com/WNL/B629).

\section{Cognitive Ability Data}

All participants in the LBC1936 underwent a detailed battery of standardized cognitive tests. From these, participant scores for 3 distinct cognitive domains (visuospatial ability, processing speed, and verbal memory) alongside a general fluid-type cognitive ability score $\left(g_{f}\right)$ were created based upon well-fitting, hierarchical structural equation models tested in our previously published work $^{30}$; relevant cognitive tests and individual weightings can be found in the supplementary document (eTable 3, links.lww.com/WNL/B629).

\section{Lifestyle Variables}

Building from previous work that looked at the impact of vascular risk factors (VRFs) on cognitive aging, ${ }^{31}$ we selected the most pertinent variables available to us in the LBC1936 cohort that may influence or confound the relationship between inflammation, brain health, and cognitive aging. Lifestyle variables included body mass index (BMI; $\left.\mathrm{kg} / \mathrm{m}^{2}\right)$, calculated from height and weight at the time of interview (see eMethods for details), alongside variables relating to selfreported health and disease history: cardiovascular disease 
Figure 2 Flowchart Depicting the Step-by-Step Selection Process of the Lothian Birth Cohort 1936 (LBC1936) Participants Included in the Final Sample for Data Analyses

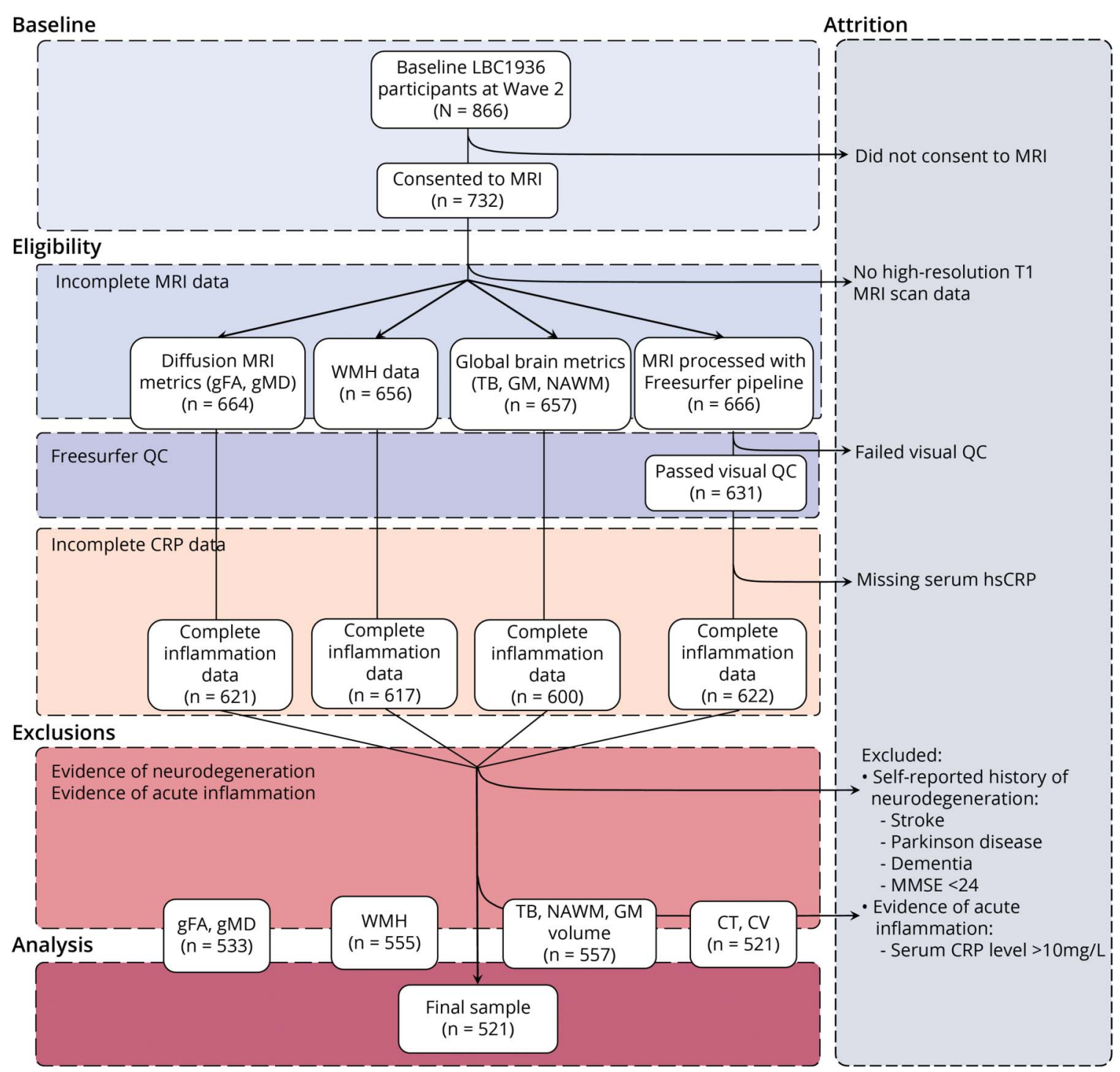

CRP = C-reactive protein; $C T$ = cortical thickness; CV = cortical volume; gFA = general fractional anisotropy; GM = gray matter; gMD = general mean diffusivity; hsCRP = high-sensitivity C-reactive protein; MMSE = Mini-Mental State Examination; NAWM = normal appearing white matter; QC = quality control; TB = total brain volume; $\mathrm{WMH}=$ white matter hyperintensities.

history (CVD), hypertension, diabetes, smoking status (coded as current smoker [1] vs ex/nonsmoker [0]), and alcohol use (coded as drinker [1] vs nondrinker [0]). Regular anti-inflammatory drug use was also collected at baseline and coded as on medication [1] or not on medication [0].

\section{Statistical Analyses}

Statistical analyses were performed in $\mathrm{R}$ version 3.6.1 (rproject.org). Alpha was 0.05 for all analyses and results were corrected for multiple comparisons using the false discovery rate (FDR). ${ }^{32}$ Standardized coefficients are reported throughout to facilitate comparison of associations. Serum measures of CRP were log-transformed to correct a positively skewed distribution. White matter hyperintensity (WMH) volume was log transformed, after which it showed an approximately normal distribution. All global MRI volumetric measures (total brain $[\mathrm{TB}]$, gray matter $[\mathrm{GM}]$, normalappearing WM [NAWM], WMH) were corrected for intracranial volume (ICV) and expressed as a ratio of ICV. For volumetric brain associations, differences between association magnitudes (serum CRP vs DNAm CRP associations) were assessed using the Williams test ${ }^{33}$ for dependent groups with overlapping correlations (cocor.indep.groups.overlaps) as implemented in the "cocor" R package (cran.r-project.org/ web/packages/cocor/cocor.pdf). We ensured that models showed acceptably low multicollinearity (variance inflation was ascertained using "vif" in the "car" package in R; cran.rproject.org/web/packages/car/car.pdf). Pairwise bivariate associations were assessed between markers of inflammation, neuroimaging, and lifestyle covariates using Pearson 
Table 1 Cross-sectional Associations Between Serum CRP and DNAm CRP With Neuroimaging and Cognitive Outcomes

\begin{tabular}{|c|c|c|c|c|c|c|c|}
\hline \multirow[b]{2}{*}{ Phenotype } & \multicolumn{3}{|c|}{ Serum CRP (model 1) } & \multicolumn{3}{|c|}{ DNAm CRP (model 2) } & \multirow{2}{*}{$\begin{array}{l}\Delta \text { Association } \\
\text { magnitudes, } p \text { value }\end{array}$} \\
\hline & $\beta$ & SE & $p$ Value & $\beta$ & SE & $p$ Value & \\
\hline \multicolumn{8}{|l|}{ Neuroimaging } \\
\hline TB & -0.033 & 0.040 & 0.579 & -0.197 & 0.041 & $8.42 \times 10^{-6 a}$ & $0.001^{a}$ \\
\hline GM & -0.026 & 0.042 & 0.670 & -0.200 & 0.043 & $1.66 \times 10^{-5 a}$ & $0.001^{a}$ \\
\hline NAWM & -0.027 & 0.042 & 0.657 & -0.150 & 0.043 & $0.001^{a}$ & $0.015^{a}$ \\
\hline WMH & 0.018 & 0.042 & 0.740 & 0.108 & 0.043 & $0.017^{a}$ & 0.069 \\
\hline gFA & -0.055 & 0.045 & 0.347 & -0.162 & 0.045 & $6.97 \times 10^{-4 a}$ & $0.032^{a}$ \\
\hline gMD & 0.025 & 0.045 & 0.677 & 0.124 & 0.045 & $0.010^{a}$ & $0.049^{a}$ \\
\hline \multicolumn{8}{|l|}{ Cognitive } \\
\hline Visuospatial ability & -0.082 & 0.038 & 0.069 & -0.097 & 0.037 & $0.014^{a}$ & 0.213 \\
\hline Processing speed & -0.088 & 0.038 & 0.054 & -0.144 & 0.038 & $4.64 \times 10^{-4 a}$ & 0.267 \\
\hline Verbal memory & -0.046 & 0.038 & 0.347 & -0.095 & 0.038 & $0.017^{a}$ & 0.175 \\
\hline$g_{f}$ & -0.098 & 0.038 & $0.027^{a}$ & -0.158 & 0.037 & $6.55 \times 10^{-5 a}$ & 0.732 \\
\hline \multicolumn{8}{|c|}{$\begin{array}{l}\text { Abbreviations: CRP = C-reactive protein; DNAm = DNA methylation; } g_{f}=\text { general cognitive ability; } \text { gFA = general fractional anisotropy; GM = gray matter; } g \text { MD = } \\
\text { general mean diffusivity; NAWM = normal-appearing white matter; } \mathrm{TB}=\text { total brain; WMH = white matter hyperintensity. } \\
\text { Cross-sectional associations of inflammation measures and neuroimaging and cognitive phenotypes. All } p \text { values reported are false discovery rate corrected. } \\
{ }^{a} p<0.05 \text {. }\end{array}$} \\
\hline
\end{tabular}

correlation. All models were adjusted for age and sex. Details of individual analyses are as follows.

\section{Volumetric Brain Associations With Inflammation}

Linear regression models were used to identify the proportion of phenotypic variance explained by DNAm CRP and to determine whether this was independent of the serum CRP signal for each brain health phenotype. Logistic regressions were conducted for self-reported disease history variables with binary outcomes (disease/no disease).

\section{Regional Brain Analyses}

Localized associations between DNAm CRP score and vertexwise cortical volume, area, and thickness were performed using linear regression, controlling for age, sex, and ICV. We used the SurfStat MATLAB toolbox (math.mcgill.ca/keith/ surf stat) for Matrix Laboratory R2012a (The MathWorks, Inc.). The resulting statistical maps ( $t$-maps) were corrected for multiple comparisons using FDR with a $q$ value of 0.05 across all 327,684 vertices on the cortical surface.

\section{Sensitivity Analyses}

Ancillary mixed-effects models including interaction terms (e.g., inflammation $\times$ age, inflammation $\times$ sex, inflammation $x$ anti-inflammatory drug use) investigated whether the association of chronic inflammation with brain health outcomes was modified or confounded by age, sex, or the use of antiinflammatory medication (eTable 4, links.lww.com/WNL/ B629). Similarly, we included lifestyle and health covariates in a fully adjusted model (alongside age and sex) to determine whether individual aspects of health and lifestyle had an impact on the association of inflammation with brain health phenotypes (eTable 5, links.lww.com/WNL/B629).

\section{Mediation Analyses}

We ran mediation analyses in a structural equation modeling (SEM) framework using the R "lavaan" package (cran.r-project.org/web/packages/lavaan/lavaan.pdf). This simultaneously characterized associations among CRP, brain, and cognitive metrics, and also specifically tested the hypothesis that brain structure would partly and significantly mediate associations between measures of CRP and cognitive ability. Both single and multiple mediator models were specified (see Figure 5, A-C, as example). Single mediator models provided information on the proportion of CRP-cognitive associations attributable to individual neuroimaging metrics. By contrast, in multiple mediator models, brain structural variables were entered simultaneously as covarying mediators (see path diagram, Figure 5C). This allowed us to quantify the proportion of variance in $\mathrm{CRP}$-cognitive associations uniquely explained by each facet of brain structure (GM, NAWM, WMH, general fractional anisotropy [gFA], general mean diffusivity [gMD]). The primary estimates of interest in this study are the degree of change (mediation) in the direct path ( $c$ to $\left.c^{\prime}\right)$ between inflammation measures (DNAm CRP or serum CRP) and cognitive ability ( $g_{f}$ or processing speed or visuospatial ability or verbal memory) when the indirect path from inflammation to cognitive ability via brain structure $(\mathrm{a} \times \mathrm{b})$ is included. A significant mediation of the $\mathrm{c}$ path (to $\mathrm{c}^{\prime}$ ) is denoted by the 
statistical significance of this indirect effect. Bootstrapping was used to calculate standard errors. Multiple comparisons were corrected for by FDR correction. These mediations were rerun when accounting for self-reported health variables as covariates: in model 1, age and sex were covariates; in model 2, they were age, sex, BMI, hypertension, diabetes, smoking status, and alcohol use. To account for missing data bias, we took account of all available data, using full information maximum likelihood estimation. ${ }^{34}$ Model fit was evaluated based on root mean squared error approximation (RMSEA), the comparative fit index (CFI), the standardized root mean square residual (SRMR), and the Tucker-Lewis index (TLI). We considered a model an acceptable fit when it respected the following thresholds: RMSEA $\leq 0.05$; SRMR $\leq 0.06$; CFI $\geq 0.97$; and $\mathrm{TLI} \geq 0.95$, as recommended. ${ }^{35}$

\section{Data Availability}

The data analyzed in this study are not publicly available as it contains data that could compromise participant consent and confidentiality, but can be requested via a data access request to the Lothian Birth Cohorts research group.

\section{Standard Protocol Approvals, Registrations, and Patient Consents}

Ethical permission for the LBC1936 was obtained from the Multi-Centre Research Ethics Committee for Scotland (MREC/01/0/56) and the Lothian Research Ethics Committee (LREC/2003/2/29). Written informed consent was obtained from all participants. All necessary patient/ participant consent has been obtained and the appropriate institutional forms have been archived.

\section{Results}

\section{DNAm CRP Is Associated With Global and Regional Brain Volume}

We studied 521 eligible older adults (aged $\sim 73$ years; refer to Figure 2 and eTable 1, links.lww.com/WNL/B629) and looked at epigenetic vs serum inflammation associations across a range of neuroimaging and cognitive measures (Table 1). To index chronic inflammation, an epigenetic measure of CRP (DNAm CRP) was assembled for each participant (see Methods). The correlation between the DNAm CRP score and serum $\log (\mathrm{CRP})$ was moderate $(r=$ $0.29,95 \%$ confidence interval $[\mathrm{CI}] 0.28-0.4)$, and the DNAm CRP score showed a stronger correlation with serum CRP than any one of its composite CpGs. ${ }^{11}$

We found that higher inflammatory burden, indexed by DNAm CRP scores, associated with poor cognitive and neuroimaging brain health outcomes (Table 1). DNAm CRP exhibited significantly larger (6.4-fold, on average) associations with brain structural MRI metrics (including global GM and WM atrophy, poorer WM microstructure, and increased WMH burden) than serum CRP. These DNAm CRP-associated brain structural changes were independent of anti-inflammatory drug use, age, or sex (eTable 4, links.lww.com/WNL/B629).
Participants with a higher inflammatory burden on average had greater overall brain atrophy, with higher DNAm CRP associating with lower total brain volume $(\beta=-0.197,95 \% \mathrm{CI}-0.28$ to -0.12 , $\left.p_{\mathrm{FDR}}=8.42 \times 10^{-6}\right)$, GM volume $(\beta=-0.200,95 \% \mathrm{CI}-0.28$ to $\left.-0.12, p_{\mathrm{FDR}}=1.66 \times 10^{-5}\right)$, and $\mathrm{WM}$ volume $(\beta=-0.150,95 \% \mathrm{CI}$ -0.23 to $\left.-0.07, p_{\mathrm{FDR}}=0.001\right)$. Models that included additional health and lifestyle covariates (BMI, smoking, alcohol consumption, hypertension, diabetes, and cardiovascular disease history) attenuated the relationship between DNAm CRP and brain health outcomes by up to $40 \%$ (eTable 5, links.lww.com/WNL/B629). Of these, the associations between DNAm CRP with WM measures (WMH, NAWM) were the most attenuated (34\%-40\%). Out of the lifestyle and health factors accounted for, smoking appeared to have the greatest influence on the attenuation (as illustrated in supplementary eFigure 2, links.lww.com/WNL/ B629).

After examining global brain structural alterations, we looked at specific regional cortical brain associations with higher inflammation levels. We found regional heterogeneity in the patterning of associations between CRP measures and cortical metrics: atrophy in frontal, anterior lateral, and medial temporal lobes was associated with higher DNAm CRP (Figure 3B); inflammation associations with brain cortical thickness are presented in the supplementary document (eFigure 1, links.lww.com/WNL/B629). Overall, these results emphasize that the DNAm-CRP score associates with lower cortical volume of specific brain regions (lateral and medial temporal regions of the brain), which show overlap with those of serum CRP and unique variance (Figure 3F), with DNAm CRP reflecting atrophy beyond the serum CRP score.

\section{DNAm CRP Is Associated With White Matter Microstructure in Specific White Matter Tracts} Next, we investigated whether higher DNAm CRP was related to lower WM microstructure based on global and regional diffusion MRI (dMRI) measures by looking at inflammation associations with WM tract fractional anisotropy and mean diffusivity. Whereas serum CRP-dMRI associations were null in all cases (all $p_{\mathrm{FDR}}>0.089$ ) (eTables 6-7, links.lww.com/WNL/B629), higher DNAm CRP scores predicted overall lower gFA $\left(\beta=-0.162, p_{\mathrm{FDR}}=6.94 \times 10^{-4}\right)$ and higher $\operatorname{gMD}\left(\beta=0.124, p_{\mathrm{FDR}}=0.010\right)$. For specific $\mathrm{WM}$ tracts, the strongest associations were seen for the arcuate fasciculus and uncinate fasciculus, with lower FA and higher MD with higher DNAm CRP (see Figure 4; eTables 6-7, links.lww.com/WNL/B629). For global measures of WM tract integrity (gFA, gMD), accounting for health and lifestyle covariates did not substantially alter the magnitude or significance of these associations (eTable 5, links.lww. $\mathrm{com} / \mathrm{WNL} / \mathrm{B} 629$ ); however, at the level of individual WM tracts, the relationship between DNAm CRP and FA and $\mathrm{MD}$ was attenuated when lifestyle factors were included in the models (eTables 8-9, links.lww.com/WNL/B629); this is illustrated in supplementary eFigure 3 (links.lww. com/WNL/B629). 
Figure 3 DNAm CRP Shows Stronger and More Widespread Associations With Global and Regional Brain Structure Than Serum CRP

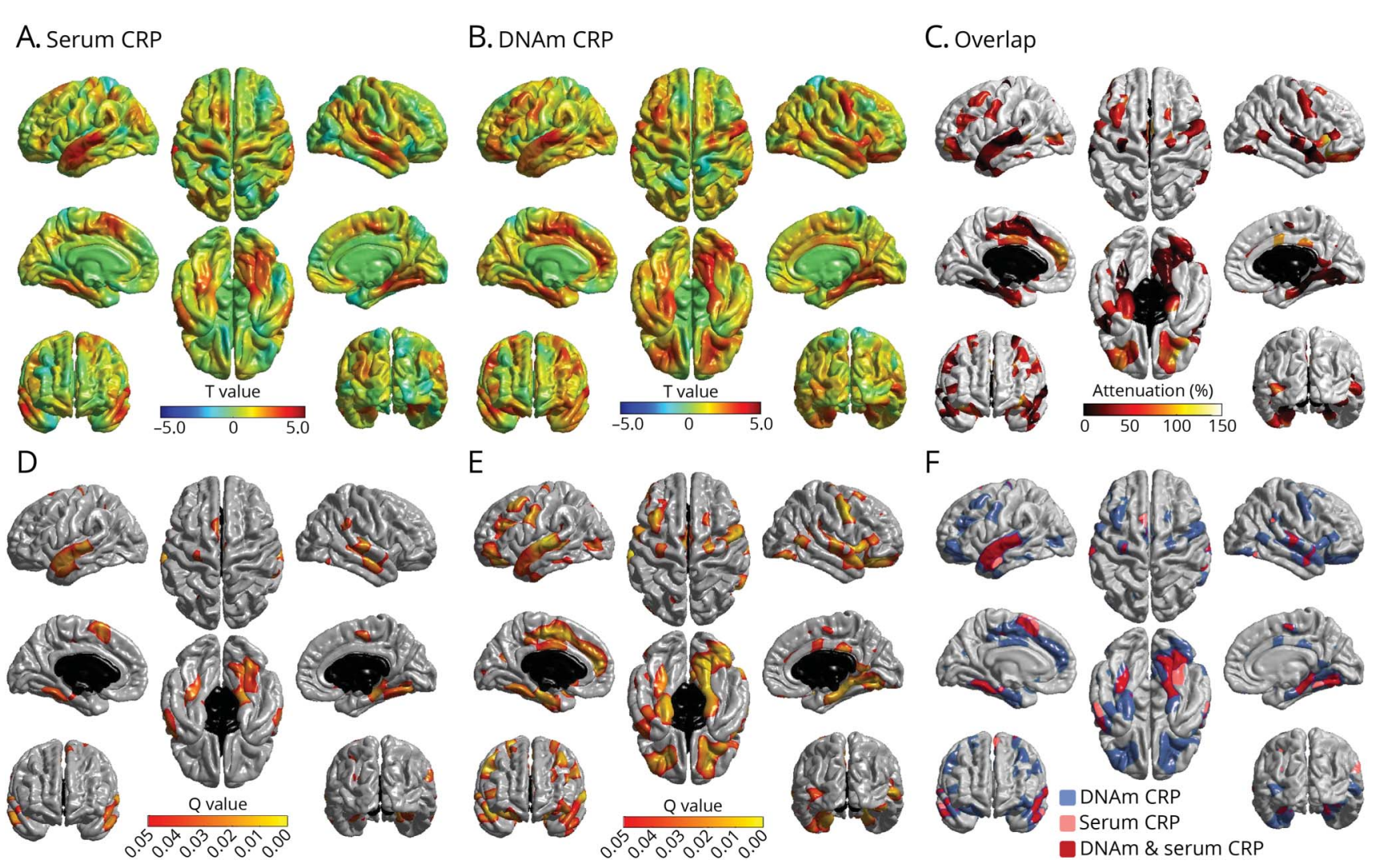

Regional cortical volume regressed against serum C-reactive protein (CRP) (A, D) and DNA methylation (DNAm) CRP (B, E) $(n=521)$. Colors denote the magnitude (T-maps; top) and significance (Q values; bottom) of the negative associations between inflammation and brain cortical volume. (C) Percentage attenuation for the significant associations between DNAm-CRP and cortical volume when also controlling for serum CRP. Conjunction plot (F) shows the spatial extent of independent contributions and overlap (red) in cortical loci that exhibit false discovery rate (FDR)-corrected unique associations with simultaneously modeled serum (pink) and epigenetic (blue) inflammation measures; results are corrected for sex, age, and intracranial volume.

\section{Brain Structure Partly Mediates the Association of DNAm CRP With Cognitive Ability}

As higher DNAm CRP levels were associated with lower cognitive performance both here (Table 1) and previously, ${ }^{11}$ we quantified the degree to which brain structural differences contribute to the inflammation-cognition association, and which facets show the strongest unique contributions to this relationship using an SEM framework. Bivariate associations between all variables (inflammation, brain structure, cognitive ability, and lifestyle measures) are provided in eTable 10 (links.lww.com/WNL/B629). Whereas TB volume, GM volume, NAWM volume, and WMH volume all emerged as significant mediators in single SEM models (percentage attenuation 14\%-21\%; eTable 11, links.lww.com/WNL/ B629), multiple mediator models were used to test the degree to which each global MRI metric contributed uniquely to mediation of the same association (Figure 5D; eTable 12, links.lww.com/WNL/B629). Here, the sum total of MRI measures significantly mediated the association between DNAm CRP and general cognitive ability $(\beta=-0.047$ $[-0.076$ to -0.018$], p_{\mathrm{FDR}}=0.002$; percentage attenuation 29.7\%). The unique contributions to this variance were largest for NAWM volume $\left(\beta=-0.03[-0.053\right.$ to -0.023$], p_{\mathrm{FDR}}=$ 0.012 ), indicating that the loss of WM may contribute to inflammation-associated differences in cognitive functioning in older age. Out of the individual cognitive domains, processing speed was the most significantly mediated by the sum total of MRI metrics $\left(\beta=-0.058[-0.090\right.$ to -0.027$]$, $p_{\mathrm{FDR}}=$ 0.001 ; percentage attenuation $41 \%)$. Again, NAWM emerged as the largest unique contribution to this variance $(\beta=-0.037$ $[-0.053,-0.007], p_{\mathrm{FDR}}=0.006$; eTable 13 [links.lww.com/ WNL/B629]). Similarly, visuospatial ability was significantly mediated by the sum total of MRI metrics $(\beta=-0.036$ $[-0.063$ to -0.010$], p_{\mathrm{FDR}}=0.013$; percentage attenuation $37 \%)$, with NAWM accounting for the largest unique contribution to this effect $\left(\beta=-0.030[-0.063\right.$ to -0.010$], p_{\text {FDR }}=$ 0.012 ). While verbal memory was significantly mediated by the sum total of MRI metrics ( $\beta=-0.031$ [ -0.057 to -0.007$]$, $p_{\text {FDR }}=0.026$; percentage attenuation $\left.33 \%\right)$, there were no significant contributions from individual MRI metrics (eTables 12-13, links.lww.com/WNL/B629).

Finally, with the addition of lifestyle and health covariates to our models, no aspect of brain structure remained a significant mediator of the associations between DNAm CRP and 
Figure 4 DNA Methylation C-Reactive Protein is Associated With White Matter Microstructure in Specific White Matter Tracts

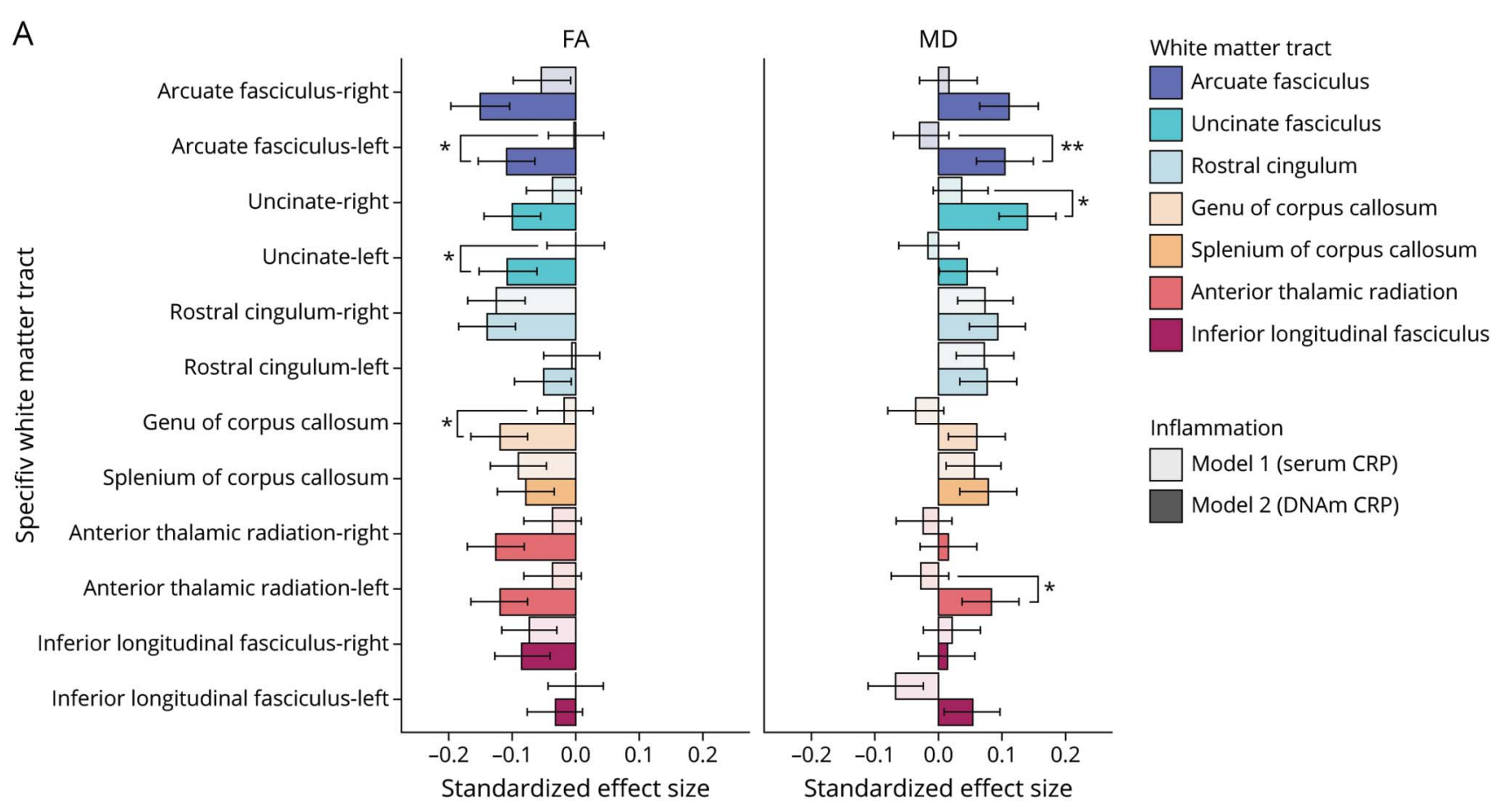

B
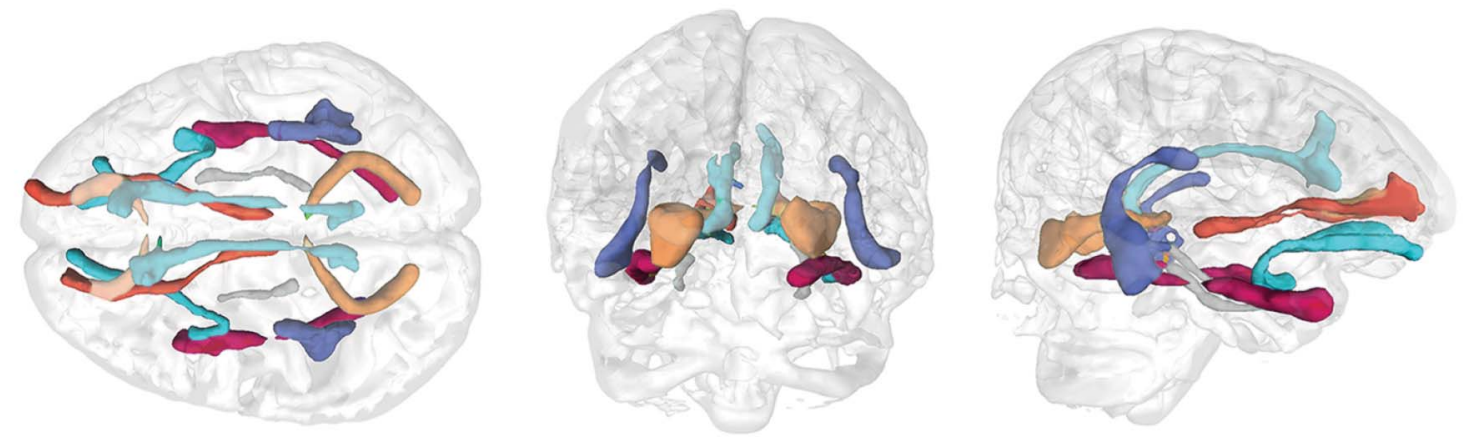

(A) Standardized regression coefficients for associations between white matter tract-averaged fractional anisotropy (FA; left) and mean diffusivity (MD; right). Bars show standardized coefficients and standard errors. Asterisks indicate where associations are significantly larger for DNA methylation than for serum $\left({ }^{*} p<0.05,{ }^{* *} p<0.01\right)$. (B) Illustration of the respective white matter tracts measured using probabilistic neighborhood tractography in one LBC1936 study participant.

general cognitive ability $\left(\beta_{\text {mediation }}=-0.023[-0.049\right.$ to 0.003 ], $p_{\mathrm{FDR}}=0.167$ (see Figure 5, eTables 11-12, links.lww. com/WNL/B629) or any of the individual cognitive domains (eTable 12, links.lww.com/WNL/B629).

\section{Discussion}

Only recently has there been a push for integrated multiomics approaches to better characterize chronic inflammation. ${ }^{3,26}$ DNAm profiles may act as promising peripheral biomarkers for cognitive-aging differences at the population level, given their relative stability in the short term, and their joint modulation by both genetic and lifestyle traits. Elsewhere, DNAm markers of inflammation have proved informative in predicting a range of age-related health outcomes, from cardiovascular disease to depression, ${ }^{23,24,36}$ but few studies have applied this same approach to cognitive aging differences in healthy cohorts. As chronic inflammation is considered to be an insidious, cumulative, and often undetected contributor to cognitive aging, ${ }^{1,3,5,14}$ the importance of such epigenetic markers may be their utility to index inflammatory load with greater reliability than phasic protein measures. In this study, DNAm CRP was more robustly associated with a range of cognitive and neuroimaging metrics than serum CRP, supporting our original hypothesis. We discovered that DNAm CRP shows consistently stronger associations with brain structure than serum CRP (on average, 6.4-fold greater), that these associations are not regionally homogeneous across the brain's cortex, and that specific aspects of brain structure partly mediate (up to $29.7 \%$ ) associations between an epigenetic signature of CRP and cognitive 


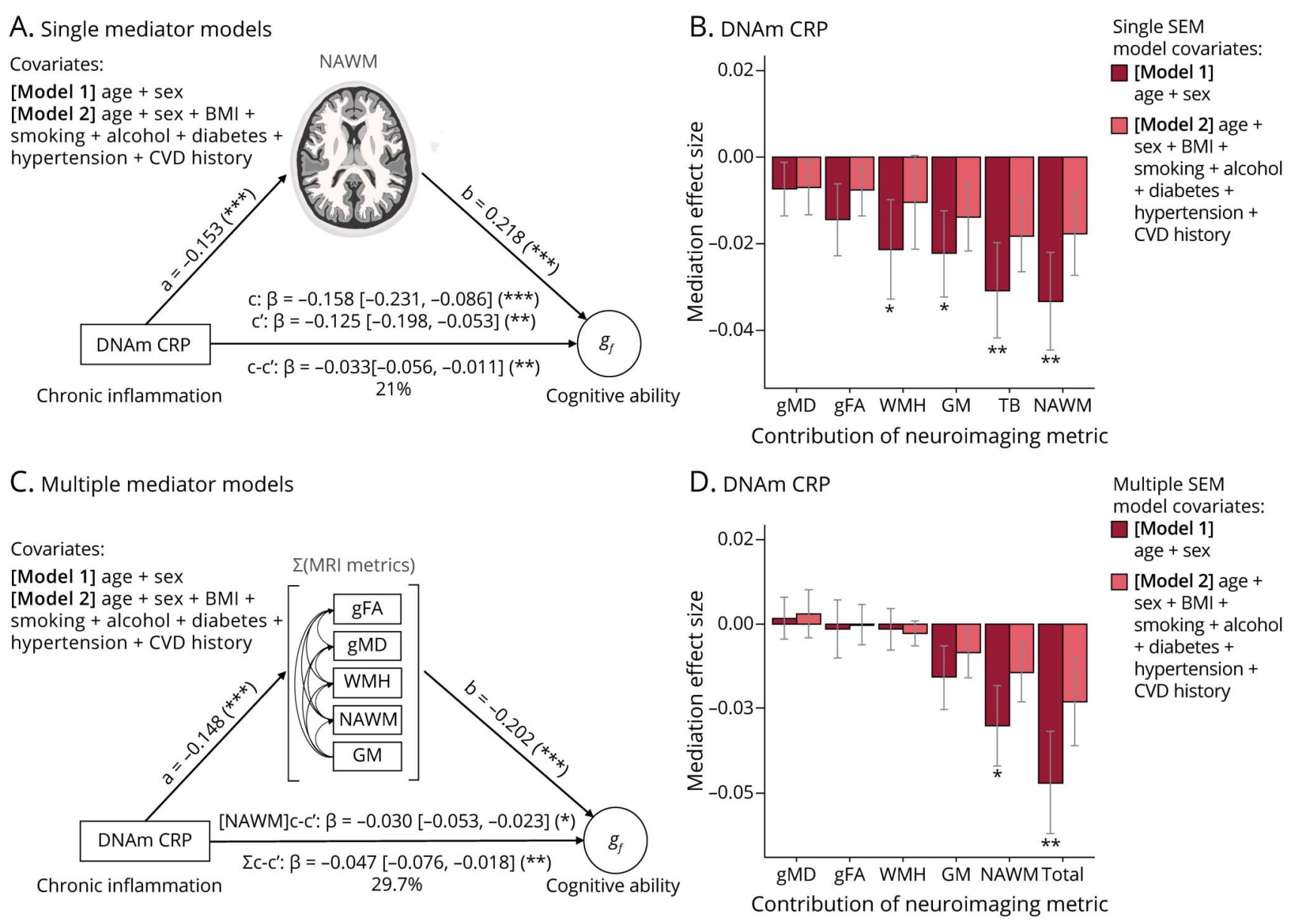

Top panel (A, B) displays single mediator models, bottom panel (C, D) displays multiple mediator models. (A) Model 1 structural equation model path diagrams showing that in model 1, the association between DNA methylation (DNAm) C-reactive protein (CRP) and general cognitive ability (path c) was significantly and partially mediated by normal-appearing white matter volume (path ab $=-0.033, p=0.001$ ), attenuating the c path by $21 \%$ (path $c^{\prime}$ ) and $(C) 29.7 \%$ by multiple MRI variables $(a b=-0.047, p=0.002$ ). (B) Single mediator models indirect effect size and standard error bars. (D) Multiple mediator models indirect effect size and standard error bars. Light bars show model 1 (includes covariates age and sex), dark bars show model 2 , which contains additional health covariates (age + sex + body mass index [BMI] + hypertension + smoking status + alcohol use + cardiovascular disease [CVD] history + diabetes); ${ }^{*} p<0.05 ; * \star p<0.01 . g_{f}=$ general cognitive ability; gFA = general fractional anisotropy; GM = gray matter; gMD = general mean diffusivity; $\mathrm{NAWM}=$ normal-appearing white matter; $\mathrm{WMH}=$ white matter hyperintensity.

functioning. Our results highlight the potential of epigenetic approaches to indexing inflammation in population cohorts and suggest that chronic inflammation may contribute to both focal and global brain structural changes that underlie differences in cognitive aging.

We found regional heterogeneity in the patterning of associations between CRP measures and cortical metrics, indicating differential regional vulnerability to chronic inflammation.

Reductions in brain cortical volume and thickness in frontal, anterior lateral, and medial temporal lobes were associated with increased DNAm CRP. Consistently, previous studies report structural changes associated with inflammatory markers in the temporal and frontal cortices. ${ }^{17,18}$ Atrophy in these regions is implicated in cognitive decline, ${ }^{37}$ and differential patterns of proinflammatory receptor distribution may underlie why some brain regions are more vulnerable to inflammation than others. For example, in patients with $\mathrm{Alz}$ heimer disease $(\mathrm{AD})$, proinflammatory cytokine receptor density and expression are increased in regions of neurodegeneration, including the medial frontal and temporal cortices. ${ }^{38}$ Higher inflammation levels have also been related to progression of atherosclerosis, with evidence for differential effects of CRP in different beds of the arterial brain supply. ${ }^{39,40}$ These findings suggest that raised levels of inflammatory mediators may contribute to localized brain atrophy via their differential expression in brain tissue and cerebrovasculature.

Overall, the results of our mediation analyses indicate that chronic inflammation's detrimental effect on WM beyond other brain structural features may underlie the inflammationassociated differences in cognitive functioning in older age. Whereas numerous studies have found associations between reduced WM volume and raised inflammatory markers in 


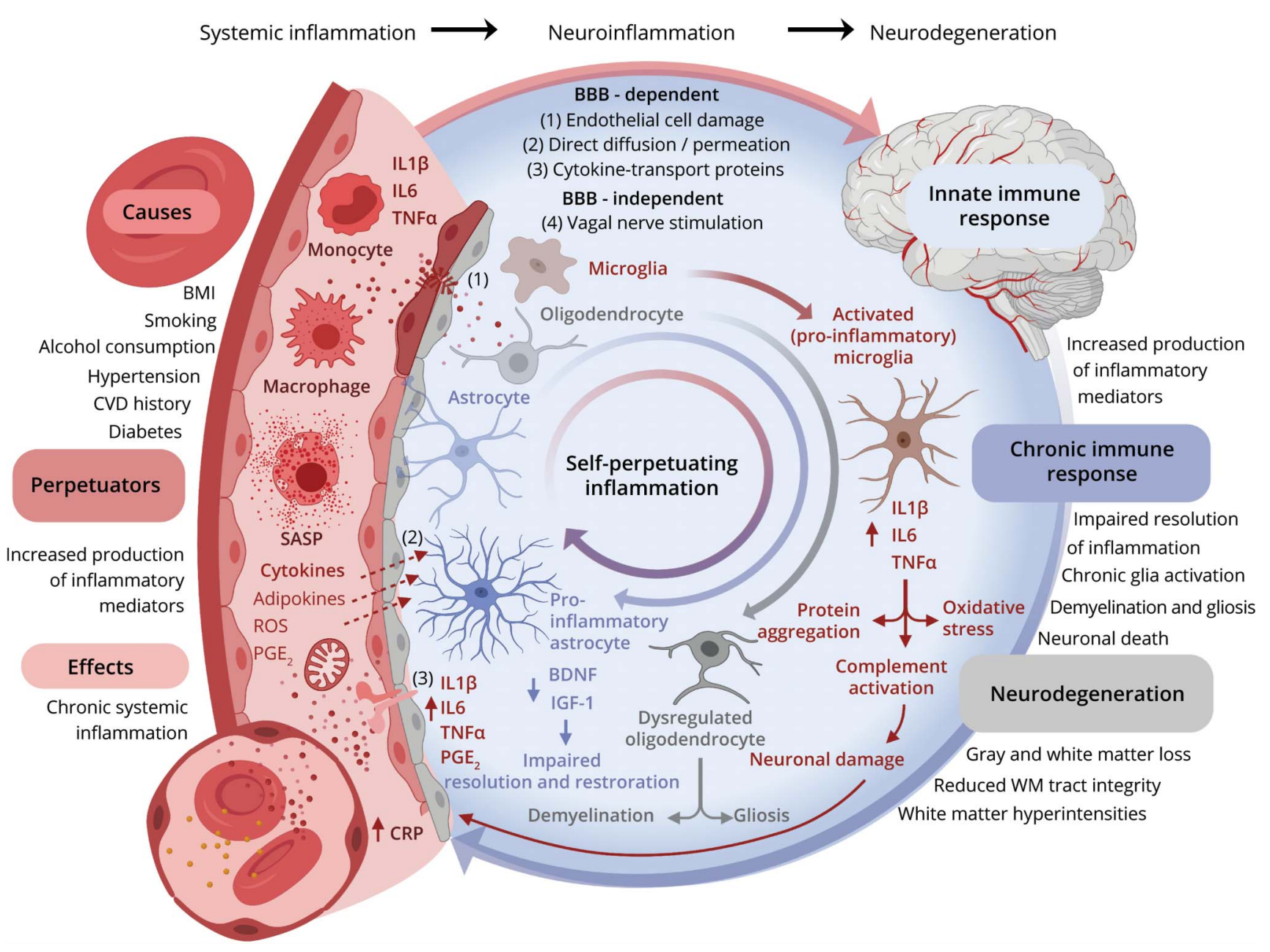

(A) Chronic inflammation is pertinent to brain aging in that inflammatory mediators in the periphery can damage the blood-brain barrier (BBB), permitting entry into the brain where they go on to disrupt neurons and glia and perpetuate a chronic inflammatory state. This directly contributes to various neurodegenerative pathways (illustrated) that lead to brain cell death. (B) Suggested mechanisms by which the causes of inflammaging (immunosenescence, lifestyle, clinical health) and related consequences may drive brain health (structural and cognitive) outcomes. (C) Study model: chronic inflammation is a key driver of cognitive decline through its effects on brain structure. Left shows generic directed acyclic graph for mediation analysis (left panel) and for the study example (right panel). $A=$ exposure; BDNF = brain-derived neurotrophic factor; $C$ = confounder; CRP = C-reactive protein; IGF-1 = insulin-like growth factor 1 ; IL1 $\beta=$ interleukin-1 $\beta$; IL6 = interleukin-6; M = mediator; PGE2 = prostaglandin E2; ROS = reactive oxygen species; SASP = senescence associated secretory phenotype; TNF- $\alpha=$ tumor necrosis factor- $\alpha ; Y=$ outcome. Created with BioRender.com.

healthy cohorts ${ }^{14,17,41}$ and those with chronic inflammatory conditions, $^{42}$ few have looked at inflammation, brain structure, and cognitive function concurrently. ${ }^{13}$ Fewer still have attempted to more robustly characterize chronic inflammation beyond assessing serum inflammatory protein profiles-although a notable exception comes from recent work, where the same DNAm CRP signature was found to be significantly associated with widespread reductions in WM integrity beyond that of serum CRP. ${ }^{36}$ In agreement with this study, but in an older cohort, we found that higher DNAm CRP related to increased WMH burden, reduced NAWM volume, and ostensibly poorer WM microstructure (lower FA and higher $\mathrm{MD}$ ). In particular, the WM tracts of arcuate fasciculus and uncinate fasciculus showed the most consistent significant relationships with DNAm CRP levels (across both FA and $\mathrm{MD}$ ), alongside significantly lower FA in the anterior thalamic radiation, which are consistent with studies assessing the effects of vascular risk on microstructure with advanced age. $^{31}$

In agreement with longstanding findings from neurocognitive studies - where, consistently, inflammation is more strongly associated with declines in processing speed than other cognitive domains ${ }^{9,10}$ _our results indicate that some cognitive domains (processing speed) may be more mediated by the brain structural consequences of chronic inflammation than others (verbal memory, visuospatial ability). Processing speed has been strongly linked to WM integrity at both global and regional levels ${ }^{43}$ and many of the downstream effects of neuroinflammatory processes directly affect WM integrity (to include demyelination, de-afferentation and gliosis; see Figure 6 and eFigure5, links.lww.com/WNL/B629). ${ }^{14}$ As such, chronic inflammation's contribution to diffuse and global WM loss may disproportionately affect cognitive 
functions that require the coordination of brain regions (e.g., processing speed), compared to more functionally localized ones (e.g., verbal memory). However, we have not formally compared the magnitude of these attenuations, and judge that this greater degree of attenuation is likely to be a general shared process ${ }^{30}$ plus some degree of noise, given that variance across cognitive domains is shared at the general level. ${ }^{30,44}$

The attenuation seen in inflammation-brain health associations when lifestyle factors were accounted for is to be expected given what is known about inflammation and VRFs on brain-health outcomes. ${ }^{3,31}$ Vascular inflammation is considered to be a shared mechanism linking cardiometabolic factors (to include hypertension and smoking) with poor cognitive outcomes. ${ }^{31,40,45}$ Figure 6 models this relationship and illustrates how increased inflammation in the periphery can result in neurodegenerative processes via BBB-dependent and independent pathways. ${ }^{46}$ The regional areas of brain loss that were particularly associated with DNAm CRP are also areas where others have shown increased BBB leakage in persons at risk of $\mathrm{AD} .{ }^{47}$ Peripheral markers of inflammation such as CRP have been consistently linked to the risk of cerebrovascular and cardiovascular events, ${ }^{1,3,5,40}$ and there is evidence that atherosclerotic and thrombotic presentations signify a chronic inflammatory process. ${ }^{20,39}$ The methylation CRP score was more strongly associated with modeled VRFs (hypertension, CVD history, diabetes, alcohol consumption, and smoking) than serum CRP (eTable 10, links.lww.com/ WNL/B629). Given that the $7 \mathrm{CpGs}$ that make up the DNAm CRP score reside in inflammation and vascularrelated genes (see eTable 2, links.lww.com/WNL/B629), these DNAm CRP-brain MRI associations may be capturing the effect of upstream inflammatory activity beyond that of serum CRP levels, which may explain why some VRFs show greater association with the DNAm score. Upstream inflammatory cytokines such as interleukin- 6 and tumor necrosis factor- $\alpha$ have been shown to be associated with increased risk of dementia where serum CRP levels showed no association, such as in the Rotterdam Study, ${ }^{6}$ Whitehall II longitudinal cohort study, ${ }^{12}$ and Framingham Study. ${ }^{8}$ Variation and measurement error in serum CRP levels may be confounding the relationship between chronic inflammation and related health and lifestyle triggers and exacerbators. ${ }^{20,21}$ Our results add to the evidence base that DNAm-based predictors of inflammation may act as a quantifiable archive of the longitudinal effects of these exposures ${ }^{11,27,36}$ and other unaccounted for health and genetic profiles that serum CRP levels fail to capture.

Strengths of this study include the large sample size, array of multimodal data, and that inflammation, lifestyle, cognitive, and structural brain variables were measured in the same individuals at about the same time. Compared to recent EWAS-neuroimaging research, this study is exceptionally well-powered with 521 participants after exclusions. ${ }^{48}$ Alongside the narrow age range, the homogeneity of the LBC1936 cohort (e.g., all participants are of Scottish ancestry) may have minimized any potentially strong confounding effects that factors such as mixed ethnicity and geography might have had in a more heterogenous sample. This cross-sectional nature means we are unlikely to capture the effect of more age-related changes in inflammatory profile and cognitive decline. Although we endeavored to remove participants with cognition-related pathology, these were screened via self-reported diagnoses, and we may be missing undiagnosed or subclinical incident neurodegenerative pathology. Similarly, while we have identified a range of health and lifestyle variables that could influence inflammatory load (BMI, diabetes, CVD history, smoking, alcohol consumption, hypertension), there are many nonmodeled variables that could contribute to this effect, as discussed in depth elswhere. ${ }^{1,3}$

Finally, a clear limitation of the study is that our epigenetic surrogate of inflammation was measured in blood rather than brain tissue. While brain-based biomarkers are the optimal choice for investigating cognitive outcomes, it is impractical to profile such methylomes in brain tissue in living humans. Furthermore, the use of postmortem brain tissue samples has its own problems (in particular, the stability of global DNAm following death ${ }^{49}$ ) and cannot reliably reflect the plastic state of methylomes in vivo. Future studies should consider examining a wider range of DNAm inflammatory markers (DNAm levels of interleukins, prostaglandins, and neurotrophins); DNAm inflammatory markers in younger participants (where there is likely greater variation in baseline inflammation levels); DNAm inflammatory markers in specific brain pathology cases (e.g., multiple sclerosis); as well as how peripheral inflammatory and neuroinflammatory DNAm patterns equate, and how each relates to cellular differences within the brain to give rise to the structural alterations we observe here. ${ }^{50}$

Our findings do not establish causality but support the hypothesis that chronic systemic inflammation may contribute to neurodegenerative brain changes that underlie differences in cognitive ability in later life. Previous studies exploring this relationship may underestimate the brain and cognitive sequelae of chronic inflammation by relying on single measurements of phasic serum proteins. By using an epigenetic inflammation measure, which integrates information from multiple immune-related $\mathrm{CpG}$ sites, we may provide a more reliable measure of chronic inflammation and thus a more comprehensive overview of the consequences of chronic inflammation on brain structure and function. Reliable monitoring of inflammatory exposure could enable clinicians to review the efficacy of drug and lifestyle interventions to attenuate inflammation levels with a view to improving cognitive outcomes.

\section{Acknowledgment}

The authors thank the Lothian Birth Cohort 1936 members who took part in this study and the Lothian Birth Cohort 1936 research team members who collected, entered, and checked data used in this article. The Lothian Birth Cohort 1936 study 
acknowledges the financial support of NHS Research Scotland through Edinburgh Clinical Research Facility.

\section{Study Funding}

This research was funded in whole or in part by the Wellcome Trust (grant number 108890/Z/15/Z). For the purpose of open access, the author has applied a CC-BY public copyright license to any author-accepted manuscript version arising from this submission. The LBC1936 and this research are supported by Age UK (Disconnected Mind project) and by the UK Medical Research Council (G0701120, G1001245, MR/M013111/1, MR/K026992/1). Methylation typing was supported by the Centre for Cognitive Aging and Cognitive Epidemiology (Pilot Fund award), Age UK, The Wellcome Trust Institutional Strategic Support Fund, The University of Edinburgh, and The University of Queensland. The Olink Proteomics assays were supported by a NIH research grant R01AG054628. This work was in part conducted in the Centre for Cognitive Aging and Cognitive Epidemiology, which is supported by the Medical Research Council and Biotechnology and Biological Sciences Research Council (MR/K026992/1) and which supports IJD. E.L.S.C. and A.J.S. are supported by funding from the Wellcome Trust 4-year $\mathrm{PhD}$ in Translational Neuroscience (108890/Z/15/Z to E.L.S.C.; $203771 / Z / 16 / Z$ to A.J.S). M.E.B., I.J.D., and S.R.C. are also supported by an NIH research grant R01AG054628. S.R.C is supported by a Sir Henry Dale Fellowship jointly funded by the Wellcome Trust and the Royal Society (grant number 221890/Z/20/Z).

\section{Disclosure}

The authors report no disclosures relevant to the manuscript. Go to Neurology.org/N for full disclosures.

\section{Publication History}

This manuscript was prepublished in MedRxiv (doi: doi.org/ $10.1101 / 2020.10 .08 .20205245)$. Received by Neurology May 11, 2021. Accepted in final form October 15, 2021.

Appendix Authors

\begin{tabular}{|c|c|c|}
\hline Name & Location & Contribution \\
\hline $\begin{array}{l}\text { Eleanor L.S. } \\
\text { Conole, BSc, } \\
\text { MRes }\end{array}$ & $\begin{array}{l}\text { Lothian Birth Cohorts group, } \\
\text { Department of Psychology, } \\
\text { University of Edinburgh, UK }\end{array}$ & $\begin{array}{l}\text { Drafting/revision of the } \\
\text { manuscript for content, } \\
\text { including medical writing } \\
\text { for content; study } \\
\text { concept or design; } \\
\text { analysis or interpretation } \\
\text { of data }\end{array}$ \\
\hline $\begin{array}{l}\text { Anna J. } \\
\text { Stevenson, } \\
\text { PhD }\end{array}$ & $\begin{array}{l}\text { Centre for Genomic and } \\
\text { Experimental Medicine, } \\
\text { Institute of Genetics and } \\
\text { Cancer, University of } \\
\text { Edinburgh, UK }\end{array}$ & $\begin{array}{l}\text { Drafting/revision of the } \\
\text { manuscript for content, } \\
\text { including medical writing } \\
\text { for content; major role in } \\
\text { the acquisition of data }\end{array}$ \\
\hline $\begin{array}{l}\text { Susana } \\
\text { Muñoz } \\
\text { Maniega, PhD }\end{array}$ & $\begin{array}{l}\text { Centre for Clinical Brain } \\
\text { Sciences, University of } \\
\text { Edinburgh, UK }\end{array}$ & $\begin{array}{l}\text { Drafting/revision of the } \\
\text { manuscript for content, } \\
\text { including medical writing } \\
\text { for content; major role in } \\
\text { the acquisition of data }\end{array}$ \\
\hline
\end{tabular}

Appendix (continued)

\begin{tabular}{lll}
\hline Name & Location & Contribution \\
\hline $\begin{array}{l}\text { Sarah E. } \\
\text { Harris, PhD }\end{array}$ & $\begin{array}{l}\text { Lothian Birth Cohorts group, } \\
\text { Department of Psychology, } \\
\text { University of Edinburgh, UK }\end{array}$ & $\begin{array}{l}\text { Drafting/revision of the } \\
\text { manuscript for content, } \\
\text { including medical writing } \\
\text { for content; major role in } \\
\text { the acquisition of data }\end{array}$ \\
\hline $\begin{array}{l}\text { Claire Green, } \\
\text { MSc }\end{array}$ & $\begin{array}{l}\text { Division of Psychiatry, Royal } \\
\text { Edinburgh Hospital, } \\
\text { University of Edinburgh, UK }\end{array}$ & $\begin{array}{l}\text { Drafting/revision of the } \\
\text { manuscript for content, } \\
\text { including medical writing } \\
\text { for content }\end{array}$ \\
& &
\end{tabular}

Maria del C. Lothian Birth Cohorts group, Drafting/revision of the

Valdés Department of Psychology, manuscript for content,

Hernández, University of Edinburgh, UK including medical writing

PhD

for content; major role in the acquisition of data

Mathew A. Division of Psychiatry, Royal Drafting/revision of the Harris, PhD Edinburgh Hospital, manuscript for content, University of Edinburgh, UK including medical writing for content; major role in the acquisition of data

\begin{tabular}{ll}
\hline Mark E. & Centre for Clinical Brain \\
Bastin, DPhil & Sciences, University of
\end{tabular} Drafting/revision of the manuscript for content, including medical writing for content; major role in the acquisition of data

Joanna M. Lothian Birth Cohorts group, Drafting/revision of the Wardlaw, MD Department of Psychology, manuscript for content, University of Edinburgh, UK including medical writing for content; major role in the acquisition of data

Ian J. Deary, Lothian Birth Cohorts group, Drafting/revision of the PhD Department of Psychology, manuscript for content, University of Edinburgh, UK including medical writing for content; major role in the acquisition of data; Study concept or design

\begin{tabular}{lll}
\hline Veronique E. & UK Dementia Research & $\begin{array}{l}\text { Drafting/revision of the } \\
\text { Miron, PhD }\end{array}$ \\
$\begin{array}{ll}\text { Institute, Edinburgh Medical } \\
\text { School, University of }\end{array}$ & $\begin{array}{l}\text { manuscript for content, } \\
\text { including medical writing }\end{array}$ \\
& Edinburgh, UK & $\begin{array}{l}\text { for content; analysis or } \\
\text { interpretation of data }\end{array}$
\end{tabular}

Heather C. Division of Psychiatry, Royal Drafting/revision of the Whalley, PhD Edinburgh Hospital, manuscript for content, University of Edinburgh, UK including medical writing for content; analysis or interpretation of data

\begin{tabular}{lll}
\hline Riccardo E. & Lothian Birth Cohorts group, & Drafting/revision of the \\
Marioni, PhD & Department of Psychology, & manuscript for content, \\
& University of Edinburgh, UK; & including medical writing \\
& Centre for Genomic and & for content; study concept \\
& $\begin{array}{l}\text { Experimental Medicine, } \\
\text { Institute of Genetics and Cancer, }\end{array}$ & or design; analysis or \\
& University of Edinburgh, UK
\end{tabular}

Simon R. Cox, Lothian Birth Cohorts group, Drafting/revision of the PhD Department of Psychology, manuscript for content, University of Edinburgh, UK including medical writing for content; study concept or design; analysis or interpretation of data

\section{References}

1. Corlier F, Hafzalla G, Faskowitz J, et al. Systemic inflammation as a predictor of brain aging: contributions of physical activity, metabolic risk, and genetic risk. Neuroimage. 2018;172:118-129.

2. Walker KA, Gottesman RF, Wu A, et al. Systemic inflammation during midlife and cognitive change over 20 years: the ARIC Study. Neurology. 2019;92(11): e1256-e1267. 
3. Furman D, Campisi J, Verdin E, et al. Chronic inflammation in the etiology of disease across the life span. Nat Med. 2019;25(12):1822-1832.

4. Tucker-Drob EM. Cognitive aging and dementia: a life-span perspective. Annu Rev Dev Psychol. 2019;1(1):177-196.

5. Franceschi C, Campisi J. Chronic inflammation (inflammaging) and its potential contribution to age-associated diseases. J Gerontol A Biol Sci Med Sci. 2014;69(suppl 1):S4-S9.

6. Engelhart MJ, Geerlings MI, Meijer J, et al. Inflammatory proteins in plasma and the risk of dementia: the Rotterdam study. Arch Neurol. 2004;61(5):668-672.

7. Schmidt R, Schmidt H, Curb JD, et al. Early inflammation and dementia: a 25-year follow-up of the Honolulu-Asia aging study. Ann Neurol. 2002;52(2):168-174.

8. Tan Z, Beiser A, Vasan R, et al. Inflammatory markers and the risk of Alzheimer disease: the Framingham study. Neurology. 2007;68(22):1902-1908.

9. Gorelick PB. Role of inflammation in cognitive impairment: results of observational epidemiological studies and clinical trials. Ann NY Acad Sci. 2010;1207(1):155-162.

10. Heringa SM, van den Berg E, Reijmer YD, et al. Markers of low-grade inflammation and endothelial dysfunction are related to reduced information processing speed and executive functioning in an older population-the Hoorn study. Psychoneuroendocrinology. 2014;40:108-118.

11. Stevenson AJ, McCartney DL, Hillary RF, et al. Characterisation of an inflammationrelated epigenetic score and its association with cognitive ability. Clin Epigenetics. 2020;12(1):113.

12. Singh-Manoux A, Dugravot A, Brunner E, et al. Interleukin-6 and C-reactive protein as predictors of cognitive decline in late midlife. Neurology. 2014;83(6):486-493.

13. Marsland AL, Gianaros PJ, Kuan DCH, et al. Brain morphology links systemic inflammation to cognitive function in midlife adults. Brain Behav Immun. 2015;48: 195-204.

14. Walker Keenan A, Power Melinda C, Hoogeveen Ron C, et al. Midlife systemic inflammation, late-life white matter integrity, and cerebral small vessel disease. Stroke. 2017;48(12):3196-3202

15. Gu Y, Vorburger R, Scarmeas N, et al. Circulating inflammatory biomarkers in relation to brain structural measurements in a non-demented elderly population. Brain Behav Immun. 2017;65:150-160.

16. Satizabal C, Zhu Y, Mazoyer B, et al. Circulating IL-6 and CRP are associated with MRI findings in the elderly: the 3C-Dijon Study. Neurology. 2012;78(10):720-727.

17. Bettcher BM, Wilheim R, Rigby $\mathrm{T}$, et al. C-reactive protein is related to memory and medial temporal brain volume in older adults. Brain Behav Immun. 2012;26(1): 103-108.

18. Janowitz D, Habes M, Toledo JB, et al. Inflammatory markers and imaging patterns of advanced brain aging in the general population [Internet]. Brain Imaging Behav. 2019; 14(4):1108-1117.

19. Volanakis JE. Human C-reactive protein: expression, structure, and function. Mol Immunol. 2001;38(2):189-197.

20. DeGoma EM, French B, Dunbar RL, et al. Intraindividual variability of C-reactive protein: the multi-ethnic study of atherosclerosis. Atherosclerosis. 2012;224(1): 274-279.

21. Bower JK, Lazo M, Juraschek SP, Selvin E. Within-person variability in high-sensitivity C-reactive protein. Arch Intern Med. 2012;172(19):1519-1521.

22. Ligthart S, Marzi C, Aslibekyan S, et al. DNA methylation signatures of chronic lowgrade inflammation are associated with complex diseases. Genome Biol. 2016;17(1): 255.

23. Ventham NT, Kennedy NA, Adams AT, et al. Integrative epigenome-wide analysis demonstrates that DNA methylation may mediate genetic risk in inflammatory bowel disease. Nat Commun. 2016;7:13507.

24. Stenvinkel P, Karimi M, Johansson S, et al. Impact of inflammation on epigenetic DNA methylation: a novel risk factor for cardiovascular disease? J Intern Med. 2007; 261(5):488-499.

25. Freytag V, Carrillo-Roa T, Milnik A, et al. A peripheral epigenetic signature of immune system genes is linked to neocortical thickness and memory. Nat Commun. 2017;8(1): 15193.

26. Zaghlool SB, Kühnel B, Elhadad MA, et al. Epigenetics meets proteomics in an epigenome-wide association study with circulating blood plasma protein traits. Nat Commun. 2020;11(1):15.
27. Byun H-M, Nordio F, Coull BA, et al. Temporal stability of epigenetic markers: sequence characteristics and predictors of short-term DNA methylation variations. PLoS One. 2012;7(6):e39220.

28. Stevenson AJ, McCartney DL, Harris SE, et al. Trajectories of inflammatory biomarkers over the eighth decade and their associations with immune cell profiles and epigenetic aging. Clin Epigenetics. 2018;10(1):159.

29. Wardlaw JM, Bastin ME, Valdés Hernández MC, et al. Brain aging, cognition in youth and old age and vascular disease in the Lothian Birth Cohort 1936: rationale, design and methodology of the imaging protocol. Int J Stroke. 2011;6(6):547-559.

30. Tucker-Drob EM, Briley DA, Starr JM, Deary IJ. Structure and correlates of cognitive aging in a narrow age cohort. Psychol Aging. 2014;29(2):236-249.

31. Cox SR, Lyall DM, Ritchie SJ, et al. Associations between vascular risk factors and brain MRI indices in UK Biobank. Eur Heart J. 2019;40(28):2290-2300.

32. Benjamini $Y$, Hochberg Y. Controlling the false discovery rate: a practical and powerful approach to multiple testing. J R Stat Soc Ser B. 1995;57(1):289-300.

33. Williams EJ. The comparison of regression variables. J R Stat Soc Ser B. 1959;21(2) 396-399.

34. Enders CK, Bandalos DL. The relative performance of full information maximum likelihood estimation for missing data in structural equation models. Struct Eq Model. 2001;8(3):430-457.

35. Schermelleh-Engel K, Moosbrugger H, Müller H. Evaluating the fit of structura equation models: tests of significance and descriptive goodness-of-fit measures. Methods Psychol Res Online. 2003;8(2):23-74.

36. Green C, Shen X, Stevenson AJ, et al. Structural brain correlates of serum and epigenetic markers of inflammation in major depressive disorder. Brain Behav Immun. 2021;92:39-48.

37. Edmonds EC, Weigand AJ, Hatton SN, et al. Patterns of longitudinal cortical atrophy over 3 years in empirically derived MCI subtypes. Neurology. 2020;94(24):e2532.

38. Hampel H, Scheloske M, Haslinger A, et al. Pattern of interleukin-6 receptor complex immunoreactivity between cortical regions of rapid autopsy normal and Alzheimer's disease brain. Eur Neuropsychopharmacol. 2000;10:372-373.

39. Schmidt R, Schmidt H, Pichler M, et al. C-reactive protein, carotid atherosclerosis, and cerebral small-vessel disease: results of the Austrian Stroke Prevention Study. Stroke. 2006;37(12):2910-2916.

40. Shoamanesh A, Preis SR, Beiser AS, et al. Inflammatory biomarkers, cerebral microbleeds, and small vessel disease: Framingham Heart Study. Neurology. 2015, 84(8):825-832.

41. Wersching H, Duning T, Lohmann $\mathrm{H}$, et al. Serum C-reactive protein is linked to cerebral microstructural integrity and cognitive function. Neurology. 2010;74(13) 1022-1029.

42. Schrepf A, Kaplan CM, Ichesco E, et al. A multi-modal MRI study of the central response to inflammation in rheumatoid arthritis. Nat Commun. 2018;9(1):2243.

43. Eckert M. Slowing down: age-related neurobiological predictors of processing speed. Front Neurosci. 2011;5:25.

44. Davies G, Armstrong N, Bis JC, et al. Genetic contributions to variation in general cognitive function: a meta-analysis of genome-wide association studies in the CHARGE consortium (N=53949). Mol Psychiatry. 2015;20(2):183-192.

45. Fornage M, Chiang YA, O'Meara ES, et al. Biomarkers of inflammation and MRIdefined small vessel disease of the brain: the Cardiovascular Health Study. Stroke. 2008;39(7):1952-1959.

46. Hsuchou H, Kastin AJ, Mishra PK, Pan W. C-reactive protein increases BBB permeability: implications for obesity and neuroinflammation. Cell Physiol Biochem. 2012, 30(5):1109-1119.

47. Montagne A, Nation DA, Sagare AP, et al. APOE4 leads to blood-brain barrier dysfunction predicting cognitive decline. Nature. 2020;581(7806):71-76.

48. Wheater ENW, Stoye DQ Cox SR, et al. DNA methylation and brain structure and function across the life course: a systematic review. Neurosci Biobehav Rev. 2020;113: 133-156.

49. Sjöholm LK, Ransome Y, Ekström TJ, Karlsson O. Evaluation of post-mortem effects on global brain DNA methylation and hydroxymethylation. Basic Clin Pharmaco Toxicol. 2018;122(2):208-213

50. Stevenson AJ, McCartney DL, Shireby GL, et al. A comparison of blood and brain derived aging and inflammation-related DNA methylation signatures and their association with microglial burdens. bioRxiv 2020. doi: 10.1101/2020.11.30.404228. 


\section{Neurology}

\section{DNA Methylation and Protein Markers of Chronic Inflammation and Their Associations With Brain and Cognitive Aging}

Eleanor L.S. Conole, Anna J. Stevenson, Susana Muñoz Maniega, et al.

Neurology 2021;97;e2340-e2352 Published Online before print November 17, 2021

DOI 10.1212/WNL.0000000000012997

This information is current as of November 17, 2021

\section{Updated Information \&} Services

References

Subspecialty Collections

\section{Permissions \& Licensing}

Reprints including high resolution figures, can be found at: http://n.neurology.org/content/97/23/e2340.full

This article cites 50 articles, 10 of which you can access for free at: http://n.neurology.org/content/97/23/e2340.full\#ref-list-1

This article, along with others on similar topics, appears in the following collection(s):

\section{Class II}

http://n.neurology.org/cgi/collection/class_ii

Cognitive aging

http://n.neurology.org/cgi/collection/cognitive_aging

Cohort studies

http://n.neurology.org/cgi/collection/cohort_studies

Public health

http://n.neurology.org/cgi/collection/public_health

Volumetric MRI

http://n.neurology.org/cgi/collection/volumetric_mri

Information about reproducing this article in parts (figures,tables) or in its entirety can be found online at:

http://www.neurology.org/about/about_the_journal\#permissions

Information about ordering reprints can be found online:

http://n.neurology.org/subscribers/advertise

Neurology ${ }^{\circledR}$ is the official journal of the American Academy of Neurology. Published continuously since 1951, it is now a weekly with 48 issues per year. Copyright Copyright ( 2021 The Author(s). Published by Wolters Kluwer Health, Inc. on behalf of the American Academy of Neurology.. All rights reserved. Print ISSN: 0028-3878. Online ISSN: 1526-632X.

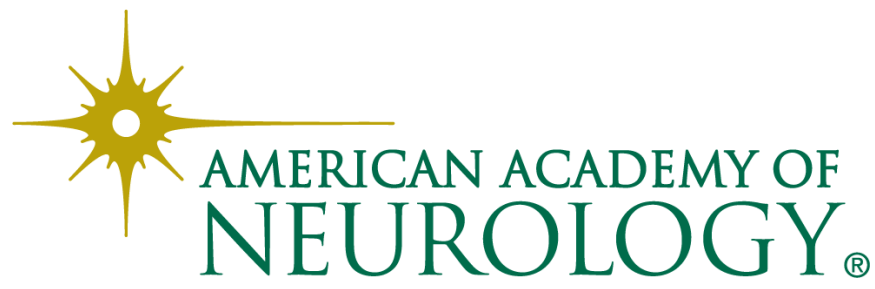

